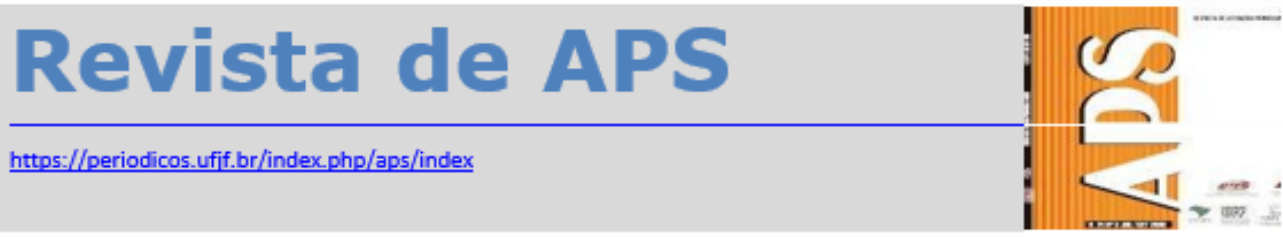

\title{
Educação interprofissional no contexto da atenção primária à saúde: relato de experiência
}

\author{
Interprofessional education in the context of Primary Health Care: \\ experience report
}

\author{
Ana Flávia de Seixas Salomão ${ }^{1}$, Ana Caroline Muzi Cunha², Jefferson Cláudio \\ Nascimento Silva ${ }^{3}$, Hianka Patrícia Cardoso Correia ${ }^{4}$, Jomara Oliveira dos Santos Yogui ${ }^{5}$, \\ Carmelita do Carmo Ribeiro Leite Camargos ${ }^{6}$, Renata Alvarenga Vieira ${ }^{7}$, Clarissa Rocha \\ Panconi Piccinini ${ }^{8}$, Sabrina Pereira Paiva ${ }^{9}$
}

\begin{abstract}
RESUMO
Introdução: A formação em saúde envolve hoje a construção e fomento de um labor colaborativo entre as profissões. O presente relato objetiva descrever e analisar as ações do projeto de extensão "Há diversidade na Unidade", desenvolvidas durante o ano de 2016. Tal projeto teve como finalidade: propiciar o desenvolvimento de competências para o trabalho interprofissional em estudantes dos períodos iniciais dos cursos de Enfermagem, Fisioterapia, Medicina e Serviço Social; inserir os estudantes em contextos reais de aprendizagem, por meio de ações em uma Unidade de Atenção Primária à Saúde de Juiz de Fora/MG; promover a integração ensino-serviço, através de ações em saúde pensadas na perspectiva interprofissional, centradas nos usuários. Desenvolvimento: Para atingir esses objetivos, a metodologia do grupo foi desenvolver, semanalmente, atividades de reconhecimento da dinâmica do território e da equipe de profissionais da Atenção Primária à Saúde. Além disso, iniciar um grupo de educação em saúde, voltado para
\end{abstract}

\footnotetext{
${ }^{1}$ Faculdade de Medicina- Universidade Federal de Juiz de Fora. E-mail: anaflaviaseixas@gmail.com.

2 Faculdade de Fisioterapia- Universidade Federal de Juiz de Fora.

${ }^{3}$ Faculdade de Serviço Social- Universidade Federal de Juiz de Fora.

${ }^{4}$ Faculdade de Enfermagem- Universidade Federal de Juiz de Fora.

${ }^{5}$ Mestre em Saúde Coletiva FAMED/UFJF. Professora de Medicina de Família e Comunidade da Faculdade de Medicina - Universidade Federal de Juiz de Fora.

${ }^{6}$ Mestre em Enfermagem/UFMG. Professora na Disciplina de Enfermagem em Saúde da Criança e do AdolescenteUniversidade Federal de Juiz de Fora.

7 Doutora em Ciências da Reabilitação/UFMG. Professora do Departamento de Fisioterapia na Saúde do Idoso, Adulto e Materno-infantil/ Universidade Federal de Juiz de Fora.

${ }^{8}$ Especialista em Ginecologia e Obstetrícia. Professora do Departamento Materno-infantil/ Universidade Federal de Juiz de Fora.

${ }^{9}$ Doutora em Saúde Coletiva UFRJ. Professora Departamento de Política e Ação do Serviço Social - Universidade Federal de Juiz de Fora.
} 
crianças, com ações planejadas de forma interprofissional. A experiência no território permitiu: ampliar a vivência dos discentes na Atenção Primária à Saúde, possibilitando a observação e reflexão sobre o trabalho em equipe nesse cenário; sensibilizar os acadêmicos para as necessidades em saúde da população; dialogar com essas necessidades, a partir do método da educação em saúde, desenvolvendo habilidades para o trabalho interprofissional. Conclusão: Os projetos de extensão podem ser uma ferramenta importante na construção da formação interprofissional em saúde, inserindo precocemente os estudantes no contexto do Sistema Único de Saúde.

PALAVRAS-CHAVE: Relações Interprofissionais. Atenção Primária à Saúde. Educação em Saúde. Relações comunidade-Instituição.

\begin{abstract}
Introduction: Nowadays, health education involves the construction and promotion of collaborative work among different professionals. The present report aims to describe and analyze the actions performed by the extension project "Há diversidade na Unidade", during the year of 2016. The goal of this project was: to encourage students from initial terms of Nursing, Physiotherapy, Medicine and Social Service to develop skills needed for interprofessional work; to introduce students to real learning contexts through actions in a Primary Health Care Unit in Juiz de Fora/MG; to promote the teaching-of-service integration using health actions based on interprofessional perspective, centered around the users of the health care services. Development: To reach these goals, the methodology included developing weekly activities to recognize the dynamics of the territory and of the primary health care professional team. Moreover, to start a health education group, designed for children, with actions planned on an interprofessional basis. The experience in the territory stimulated the students to broaden their experience in Primary Health Care by observing and reflecting about the teamwork in this scenery; to be sensitive about the population's health needs; to interact with these needs using the health education method, developing skills for interprofessional work. Conclusion: Extension projects can be an important tool for the development of health interprofessional training, inserting students earlier in the context of the Single Health System.
\end{abstract}

KEYWORDS: Interprofessional Relations. Primary Health Care. Health Education. Community-Institutional Relations.

\title{
INTRODUÇÃO
}

A educação interprofissional constitui-se como tema de destaque no campo da saúde em todo o mundo, apontando para uma ampla reforma do modelo de formação profissional. Por esse conceito, entende-se "o processo em que estudantes de duas ou mais profissões aprendem sobre os outros, com os outros e entre si para possibilitar a colaboração eficaz e melhorar os resultados na saúde." ${ }^{1: 7}$

Trata-se de um tema fundamental, a partir do momento em que se tem como 
objetivo a integralidade do cuidado no sistema de saúde. Atender às variadas dimensões das necessidades de saúde ${ }^{2}$ da população "torna a qualidade da comunicação e a colaboração entre os diferentes profissionais envolvidos no cuidado, fundamental e crítica para a resolubilidade dos serviços." 3:977 Por isso, essa nova concepção na forma de produzir cuidado vai de encontro ao isolamento entre os cursos de saúde, ressaltando a importância de criar oportunidades de educação interprofissional na formação universitária.

Nesse sentido, o processo de implementação das Diretrizes Curriculares Nacionais para os cursos da área da saúde tem revelado aspectos fundamentais, que orientam a busca por novos caminhos e referenciais de formação, através de propostas curriculares, que articulam o compromisso do processo formativo com SUS, na perspectiva da integralidade do cuidado. Isto possibilita um trabalho em saúde, que transcende os fazeres individualizados de cada profissão, assumindo a importância do trabalho coletivo. ${ }^{4}$

Considerando esses desafios, foi criado o projeto de extensão "Há diversidade na Unidade", um grupo interdisciplinar, composto por professores da Universidade Federal de Juiz de Fora (UFJF), das áreas de Enfermagem, Fisioterapia, Medicina e Serviço Social, no contexto de um curso de formação vinculado ao Pró-Saúde da UFJF (2012-2016).

Esse projeto de extensão contou com os seguintes objetivos: propiciar o desenvolvimento de competências para o trabalho interprofissional em estudantes dos períodos iniciais da área da saúde; inserir os acadêmicos em contextos reais de aprendizagem, por meio de ações em uma Unidade de Atenção Primária à Saúde de Juiz de Fora/MG; promover a integração ensino-serviço, através de ações em saúde pensadas na perspectiva interprofissional, centradas nos usuários.

Dessa forma, o presente artigo visa relatar e analisar essa experiência de formação e trabalho interprofissional, vivenciada pelos docentes e discentes do grupo, em conjunto com a equipe de saúde de uma Unidade de Atenção Primária à Saúde com Estratégia de Saúde da Família (USF) do município de Juiz de Fora/MG, destacando seus limites, desafios e possibilidades. 


\section{DESENVOLVIMENTO}

\section{Percurso metodológico}

O projeto de extensão "Há Diversidade na Unidade", com o objetivo de refletir os diversos campos da área da saúde, contou com professores-orientadores e bolsistas dos cursos de Serviço Social, Enfermagem, Fisioterapia e Medicina. O cenário de trabalho do grupo, em 2016, foi uma USF na Zona Leste de Juiz de Fora.

Para atingir os objetivos propostos pelo grupo, as atividades do projeto contaram com os seguintes eixos: 1)Ampliar o conhecimento e a vivência dos discentes sobre a Atenção Primária à Saúde, possibilitando a observação do trabalho em equipe no contexto real da Estratégia de Saúde da Família (ESF); 2)Compreender as necessidades em saúde da população do território e as determinações do processo de saúde/doença; 3)Possibilitar aos estudantes colocar em prática o trabalho interprofissional, a partir da construção de um grupo de educação em saúde, ${ }^{5}$ que dialogasse com as necessidades em saúde da população, estimulando a autonomia e a promoção de hábitos de vida saudáveis.

Nessa perspectiva, o grupo encontrou-se uma vez por semana na USF e, inicialmente, realizou atividades centradas no reconhecimento do território e da equipe de saúde. Posteriormente, constituiu-se o grupo de educação em saúde, com 20 crianças entre 5 a 11 anos, residentes na área de abrangência da USF, com periodicidade também semanal.

Além disso, o trabalho englobou uma reunião semanal, fora do cenário de prática, que objetivava construir diálogos sobre as experiências vivenciadas.

\section{A aproximação à realidade da Atenção Primária à Saúde no município de Juiz de Fora/MG}

A Atenção Primária à Saúde (APS) é constituída pelas ações e serviços que visam à promoção, prevenção, diagnóstico e prestação do cuidado em saúde. ${ }^{6}$ De acordo com - Ministério da Saúde, essas práticas devem ser orientadas por uma abordagem interprofissional e interdisciplinar, dirigida às populações em seus territórios. ${ }^{7}$ Em 1994, 
passou-se a adotar o Programa de Saúde da Família (hoje Estratégia de Saúde da FamíliaESF) como modelo estruturante dos sistemas municipais de saúde.

A rede de serviços de atenção básica em Juiz de Fora está constituída por 63 Unidades Básicas de Saúde. A região leste, que sediou o projeto, possui oito unidades, as quais atendem uma população de aproximadamente 67.351 pessoas (13\% da população atendida em Juiz de Fora). ${ }^{6}$

Além de atender uma área vasta e plural, a USF, que sediou o projeto, foi um espaço privilegiado, pois conta com agentes comunitários de saúde, assistente social, dentista, enfermeira, farmacêutica, médica, técnicas de enfermagem e a auxiliar de limpeza, criando condições favoráveis ao trabalho em equipe.

Considerando que a territorialização é um elemento estruturante do sistema de serviços de saúde e, portanto, fundamental para o entendimento da dinâmica da Atenção Primária à Saúde, a primeira ação do grupo foi conhecer a área de abrangência da USF. Buscou-se compreender a dinâmica social, econômica, política, cultural, ou seja, as características desse território. Por isso, conjuntamente às Agentes Comunitárias de Saúde (ACS), foi feito o reconhecimento do bairro e suas microáreas, incluindo as diversas instituições públicas ali instaladas, como o Centro de Referência e Assistência Social (CRAS), as escolas e, principalmente, a USF.

Nesse processo, também foi observada a condição socioeconômica dos moradores, a precariedade da infraestrutura urbana (especialmente saneamento, coleta de lixo, acesso à água tratada) e a necessidade de políticas públicas para atender direitos básicos requisitados pela comunidade, como a redução da violência urbana. Notava-se uma preocupação latente - tanto por parte dos profissionais, quanto da comunidade- com as crianças do bairro que se encontram frequentemente vulneráveis aos conflitos.

O momento de reconhecimento do território foi essencial para o grupo, já que, para a maioria, era a primeira visita àquele território, conhecido popularmente como área de vulnerabilidade social, com a presença constante das disputas territoriais entre facções criminosas e do combate com a polícia. Por isso, podia-se sentir um receio inicial, que foi, paulatinamente, reduzido com as visitas e conversas com os moradores. Assim, o grupo pôde se aproximar do contexto de vida da população e perceber os determinantes do processo saúde/doença, deixando para trás uma visão negativa e 
amedrontada da população que vive naquele bairro, a qual é usuária dos serviços públicos de saúde.

Concomitantemente, começou-se a reconhecer e a entender a dinâmica de funcionamento da unidade de saúde: sua estrutura, os serviços prestados, os profissionais envolvidos e a dinâmica do trabalho na perspectiva da ESF.

Somente, a partir da observação do trabalho da equipe de saúde, no cenário real de prática, foi possível ao grupo entender a sua complexidade e transcender a concepção simplista de que, para realizar o trabalho interprofissional, 'basta haver vários profissionais reunidos em uma mesma unidade de saúde'. Foram vivenciados diferentes exemplos de como a integralidade do cuidado em saúde depende do diálogo e colaboração entre os profissionais, que, por sua vez, só se realiza quando há entrosamento, confiança, determinação de papéis e uma liderança colaborativa.

Da mesma forma, percebeu-se que o trabalho em equipe não significa a ausência de conflitos. A USF reunia profissionais com personalidades e preferências distintas, que tiveram diferentes histórias de vida, de diferentes gerações. Por vezes, essa realidade resultava em conflitos na equipe, fazendo o grupo de extensão perceber a importância de uma liderança capaz de resolvê-los democraticamente, aproveitando as melhores qualidades e talentos de cada profissional.

Posteriormente, foram realizadas reuniões entre os membros do projeto, a gestora e as ACS, para levantar possibilidades de ações de extensão no bairro, focadas na educação em saúde e compatíveis com as necessidades da comunidade. Decidiu-se, então, iniciar um grupo, coordenado pelos próprios membros do projeto de extensão, com foco nas crianças de 5 a 11 anos.

\section{O trabalho educativo com crianças em situação de "vulnerabilidade social"}

Para realizar o trabalho educativo, foram realizadas visitas domiciliares na região próxima à USF, acompanhadas dos Agentes de Saúde, para averiguar o público-alvo e conversar com os responsáveis pelas crianças. Isso possibilitou compreender o seu contexto de vida, incluindo as condições socioeconômicas de cada família, o que foi um norteador importante no planejamento das atividades.

Os encontros, planejados e executados numa perspectiva interprofissional, 
abordaram os tópicos: higiene, alimentação saudável, meio ambiente, acidentes na infância, sonhos e autoestima e foram realizadas atividades práticas relacionadas com a higiene das mãos, higiene bucal, verificação do peso e estatura. Foram utilizadas metodologias como: colagens, brincadeiras (mímica, gincana, entre outras), desenhos, músicas e teatro.

Inicialmente o grupo foi estimulado a construir o conceito ampliado de saúde, a partir da própria percepção das crianças, estimulando que compartilhassem com o grupo seus conhecimentos prévios, opiniões e vivências familiares sobre cada assunto. Dessa forma, enriqueceu-se o diálogo e aprendizagem de todos os membros do grupo (crianças e bolsistas), a partir de uma abordagem horizontal e lúdica.

Durante o planejamento e execução das atividades, buscou-se o desenvolvimento de competências interprofissionais, tais como: clarificação de papéis da equipe executora, alternância de lideranças, comunicação e administração de conflitos. Previamente, foi definida a função de cada membro do grupo na atividade a ser desenvolvida, por exemplo: líder, responsável pela alimentação, responsável pela higienização das mãos e bucal, responsável pelos materiais e apoio, sendo que a cada semana havia alternância de funções. Eventualmente, surgiram conflitos e imprevistos que exigiam uma adequação à nova realidade.

Um dos problemas enfrentados, que exemplificam essa situação, foi a rotatividade de parte das crianças de uma semana para a outra, o que tornava relativamente imprevisível a quantidade de participantes e a faixa etária predominante. Por isso, às vezes foi preciso adquirir novos materiais para atender a todas as crianças e readequar a metodologia, o que foi feito, a partir da comunicação resolutiva entre os membros do projeto.

Também foi preciso lidar com situações, envolvendo violência entre as crianças, tanto física, como verbal. Existiram momentos em que as crianças refletiram as rivalidades existentes na comunidade, entre as famílias e territórios do bairro. Nesses casos, o papel das ACS foi fundamental para que os membros do grupo de extensão pudessem compreender os conflitos e pensar a melhor forma de contorná-los.

\section{O aprofundamento teórico como aporte para o desenvolvimento do trabalho coletivo em saúde}


Os momentos no cenário de prática foram intercalados semanalmente por reuniões de estudo e planejamento do grupo de extensão (discentes e docentes), com diferentes objetivos.

O primeiro deles foi o debate teórico, a partir de temas como Prática e Ensino Interprofissional, Educação em Saúde, conceito de necessidades em saúde e integralidade da atenção. Esses estudos contribuíram para solidificar conceitos e conhecimentos que foram aplicados durante as atividades interprofissionais na comunidade. Tratando-se de um grupo com estudantes dos primeiros períodos da graduação, essa tarefa adquiriu uma relevância ainda maior.

Assim, o grupo pôde confrontar, durante todo o projeto, as teorias sobre trabalho interprofissional e a prática real do serviço de saúde em que estavam inseridos, ou seja, a realidade das equipes no espaço de atuação, evidenciando-se a complexidade e subjetividades das relações, que só pôde ser compreendida a partir da vivência.

Em muitas reuniões do grupo, o foco foi a preparação das atividades de educação em saúde com as crianças. O desenvolvimento das atividades exigiu não apenas o conhecimento sobre educação em saúde, mas também sobre métodos pedagógicos, para se trabalhar com esse público-alvo. Contudo, foi exatamente esse contexto, que possibilitou uma vivência não apenas entre profissionais da área da saúde, mas também pedagogos e graduandos de Psicologia consultados pelo grupo, que auxiliaram na elaboração de métodos e estratégias adequadas à finalidade desejada.

\section{CONSIDERAÇÕES FINAIS}

Diante do relato apresentado, é possível perceber que o Projeto “Há Diversidade na Unidade" aprimorou a formação dos estudantes da área da saúde e contribuiu para a formação de profissionais mais dispostos e aptos ao trabalho em equipe. A vivência nos espaços reais de trabalho do SUS permitiu que os acadêmicos problematizassem concepções simplistas sobre como trabalhar em conjunto com outros profissionais e avançassem na compreensão do papel das diferentes formações.

Concomitantemente, a inserção precoce dos estudantes nos cenários da APS, de forma orientada e supervisionada, permitiu a consolidação de conceitos teóricos trabalhados nos cursos da graduação, como territorialização, necessidades em saúde, 
integralidade, educação em saúde. E, além disso, possibilitou a reflexão sobre préconceitos e julgamentos que os estudantes trouxeram consigo, ao ingressar na universidade, sobre a APS e o trabalho nas comunidades periféricas. Ao conversar e aprender com os usuários, conhecer suas experiências, construir vínculos, iniciou-se a ruptura de um modelo de formação de profissionais de saúde que não dialogam entre si, nem com a realidade social do país.

Em meio às habilidades interprofissionais trabalhadas, destaca-se o vínculo e o afeto construído entre os membros do grupo e deles com as crianças, o que tornou possível a construção de um ambiente propício à troca entre os diversos saberes, onde todos se sentiam parte do mesmo processo de educação em saúde. Ressalta-se, dessa forma, como a atuação interprofissional permite uma aproximação à perspectiva da integralidade e humanização do cuidado; mas, ao mesmo tempo, requer competências e treinamento específicos, assim como planejamento das ações.

Dessa forma, percebe-se que os projetos de extensão podem ser uma ferramenta importante para proporcionar tais experiências interprofissionais aos estudantes, inserindo-os nos cenários da APS e do SUS. Contudo, deve-se ressaltar a importância de desenvolver essas ações, desde o começo da formação, no intuito de construir outra concepção sobre as formas de produzir cuidado em saúde, que privilegie a colaboração entre profissionais e o bem-estar dos usuários.

\section{REFERÊNCIAS}

1. Organização Mundial da Saúde. Marco para Ação em Educação Interprofissional e Prática colaborativa. Gabinete da Rede de Profissões de Saúde - Enfermagem \& Obstetrícia do Departamento de Recursos Humanos para a Saúde. Genebra: OMS; 2010.

2. Cecílio LCO. As necessidades de saúde como conceito estruturante na luta pela integralidade e equidade na atenção em saúde. In: Pinheiro R, Mattos RA (Org.). Os sentidos da integralidade na atenção e no cuidado de saúde. 8 a ed. Rio de Janeiro: IMS-UERJ/Abrasco; 2009. p.117-130.

3. Peduzzi M, Norman IJ, Germani ACCG, Silva JAM, Souza GC. Educação interprofissional: formação de profissionais de saúde para o trabalho em equipe com foco nos usuários. Rev. Esc. Enferm. USP. 2013; 47(4):977-83.

4. Batista NA. Educação interprofissional em saúde: concepções e práticas. Caderno FNEPAS. 2012; 2(2):25-8. 
5. Stotz EN. Enfoques sobre educação e saúde. In: Valla VV, Stotz EN (Org.).

Participação popular, educação e saúde: teoria e prática. 1a ed. Rio de Janeiro:

Relume-Dumará; 1993. p. 11-22.

6. Juiz de Fora, Secretaria de Saúde. Plano Diretor de Atenção Primária à Saúdeprojeto de implantação. Juiz de Fora; 2014. Disponível em: <https://www.pjf.mg.gov.br/secretarias/ss/plano_diretor/docs/implantacao.pdf.

7. Brasil. Ministério da Saúde. Política Nacional de Atenção Básica. Brasília: Ministério da Saúde; 2011.

Submissão: abril de 2017.

Aprovação: agosto de 2017. 